\title{
Infering trust in web-based social networks: an analysis from TidalTrust and T-SWEETS algorithms
}

\author{
Edeilson Milhomem Silva ${ }^{1}$, Diego Oliveira Rodrigues ${ }^{2}$, Ana Carolina Salgado ${ }^{3}$ e Silvio Romero \\ Lemos Meira $^{3}$ \\ ${ }^{1}$ Federal University of Tocantins - UFT, Department of Computer Science, Palmas-TO, Brazil \\ ${ }^{2}$ University of Campinas - UNICAMP, Institute of Computing, Campinas-SP, Brazil \\ ${ }^{3}$ Federal University of Pernambuco - UFPE, Informatics Center, PE, Brazil \\ Data de recebimento do manuscrito: 28/02/2020 \\ Data de aceitação do manuscrito: 03/03/2020 \\ Data de publicação: 09/03/2020
}

\begin{abstract}
This work presents an analysis between two approaches for inferring trust in social networks: TidalTrust and T-SWEETS. The first part of this research is composed by an experiment with the Epinions, Slashdot and Wikipedia datasets in order to define the most suitable type of trust value (integer or fractioned, positive or negative, and so on). Furthermore, the second part of this research reports a comparative analysis between both algorithms. The results indicate that T-SWEETS outperforms TidalTrust in terms of accuracy and maintains the transitivity principle, which is the basic principle of trust.
\end{abstract}

Keywords - trust inference, social network, recommender systems

\section{INTRODUÇÃO}

C onfiança oferece subsídios para apoiar a sociedade em tomadas de decisões, que vão desde as mais básicas até as mais complexas - é um dos elementos que mantém a sociedade [1]. O conceito de confiança é objeto de pesquisas em diferentes áreas de conhecimento, por exemplo, Psicologia [2, 3], Sociologia [4], Filosofia [5] e Computação [6, 7]. Um trabalho pioneiro sobre confiança em ambientes computacionais foi o desenvolvido por Marsh [4], que concebeu um modelo para aplicação em sistemas de Inteligência Artificial.

As plataformas virtuais sociais possuem uma estrutura que habilita pesquisas avançadas para a identificação de relacionamentos de confiança entre indivíduos. Há três elementos que estão relacionados à confiança que são fortemente discutidos na literatura, de acordo com Reis [8], são eles, subjetividade, contexto e dinamismo.

I. subjetividade: confiança é subjetiva e, portanto, assimétrica, isto é, a confiança de uma pessoa $\mathrm{p}$, em relação a uma pessoa p1 não é a mesma confiança da pessoa $\mathrm{p} 1$ em relação a p;

II. contexto: confiança depende do contexto, ou seja, pode ser medida de uma pessoa em relação à outra, em um

Dados de contato: Edeilson Milhomem Silva, Quadra 109 Norte, Avenida NS-15, ALCNO-14, Plano Diretor Norte, Palmas/TO, Brazil, edeilson.milhomem@uft.edu.br grupo ou até mesmo diante de uma determinada situação;

III. dinamismo: confiança é dinâmica, isto é, muda com o tempo - pode aumentar se as experiências forem positivas, e pode diminuir se as experiências forem negativas.

Assim, no âmbito computacional, para a aplicação em redes sociais, Golbeck [7] define algumas propriedades de "confiança": transitividade, agregabilidade e assimetria. A transitividade representa a possibilidade de transferir a confiança de pessoa a pessoa. A agregabilidade representa a condição que define se uma pessoa procurará informações adicionais para decidir se confia ou não em outra pessoa. Já a assimetria, indica que o nível de confiança de uma pessoa em relação à outra é unilateral.

Nesta perspectiva, diversos algoritmos foram propostos para a inferência de relacionamentos ocultos de confiança em ambientes virtuais sociais, tais como, TidalTrust [9]; o RNTrust, [10]; SUNNY [11, 12]; FlowTrust [8]; o de Mamami [9] e o T-SWEETS [13]. Neste sentido, o presente trabalho tem como objetivo apresentar uma análise do algoritmo TSWEETS em relação ao algoritmo clássico TitalTrust. A análise é baseada nos resultados apresentados no trabalho de Golbeck [9] e fontes de dados de relacionamentos de confiança oriundos do Epinions ${ }^{1}$, Slashdot ${ }^{2}$ e Wikipedia ${ }^{3}$.

\footnotetext{
${ }^{1}$ Epinions.com: Product Reviews and Consumer Reports: http://www.epinions.com/

${ }^{2}$ Slashdot: News for nerds, stuff that matters: http://slashdot.org/

${ }^{3}$ Wikipédia, a enciclopédia livre: https://pt.wikipedia.org/
} 


\section{TIDALTRUST E T-SWEETS}

Para que o algoritmo TidalTrust possa realizar a inferência dos relacionamentos de confiança entre os indivíduos, inicialmente, faz uma seleção dos nós que serão considerados na análise. Nesta seleção são estabelecidos o tamanho máximo dos caminhos e os valores mínimos de confianças entre os nós que poderão ser explorados durante a análise de confiança. Em seguida, com estes valores é possível excluir alguns nós, os considerados caminhos menos irrelevantes, que segundo Golbeck [9], reduziriam a precisão da análise de confiança.

T-SWEETS, um algoritmo desenvolvido por nós [13] para inferência de confiança em ambientes virtuais sociais [13], baseia-se em 4 (quatro) elementos para inferência destes relacionamentos de confiança, são eles: similaridade entre perfis: considera as pessoas que possuem perfis similares; relacionamento de confiança: considera as relações de confiança de um usuário a em relação a um usuário b atribuída explicitamente por $\mathrm{a}$; ou a probabilidade de a confiar em $\mathrm{b}$ dado que existe um caminho de relacionamento de confiança (e. g.) entre eles; nível de maturidade: representa o quanto as produções dos usuários são bem avaliadas por outros usuários na rede social; e reputação: é a visão que um grupo de pessoas $g$ tem sobre uma pessoa $p$, determinada pelo grau de confiança que $g$ tem em relação à $p$.

Destes elementos, o Relacionamento de Confiança, será objeto de estudo neste trabalho, já que é o elemento que baseia-se em características similares ao algoritmos clássico da literatura, TidalTrust [9]. Este elemento baseia-se nas propriedades de confiança definidas por Golbeck [7], que são: Transitividade, Assimetria e Agregabilidade.

\section{EXPERIMENTO}

\section{a. Objetivo}

Para a análise da eficácia do algoritmo T-SWEETS, inicialmente, foi realizado um experimento considerando os resultados obtidos nos relacionamentos de confiança oriundos do trabalho desenvolvido por Leskovec [14]; e depois foi realizada uma análise em relação ao algoritmo TidalTrust [9].

\section{b. Cenário}

Foram utilizadas como fonte de relacionamentos de confiança, dados oriundos do Epinions, Slashdot e Wikipedia. Epinions é um ambiente em que os usuários podem realizar avaliações a determinados produtos (e.g. notebook e smartphone) ou avaliar pessoas (os próprios avaliadores). A partir das avaliações realizadas entre estes avaliadores forma-se uma rede social de relacionamentos de confiança. Slashdot, um site voltado para publicações de notícias de tecnologia. Neste ambiente, os usuários podem associar tags a outros usuários classificando-os como amigos ou inimigos. Wikipedia, uma enciclopédia online em que as pessoas podem colaborar livremente para o seu desenvolvimento. Os usuários podem passar para a categoria de administradores da enciclopédia, a partir das avaliações que podem receber de outros usuários, que são positivas, negativas e neutras. Estas fontes de dados são oriundas do trabalho de Leskovec [14], e estão disponíveis no projeto SNAP4, desenvolvido

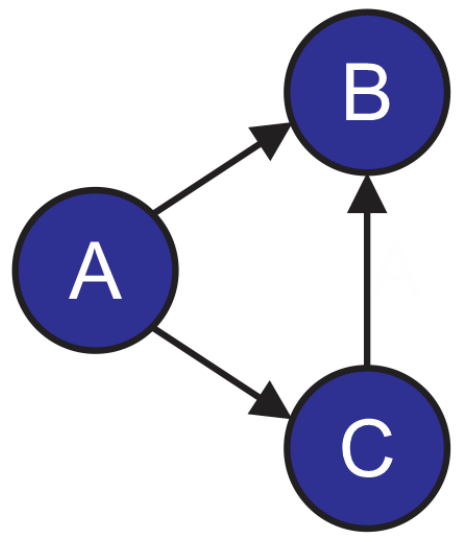

Figura 1: Estrutura de uma tríade

por pesquisadores de Stanford. O trabalho de Leskovec [14] analisa estes relacionamentos a partir de associações ternárias (tríades), que são relacionamentos formados pelo conjunto de três nós, conforme apresentado na Figura 1.

Cada relacionamento forma uma relação de confiança de valores $\{-1,+1\}$, em que o valor positivo indica que há a confiança entre os nós, enquanto o valor negativo indica a ausência de confiança. De acordo com este cenário, a Tabela 1 apresenta a proporção dos dados originados de cada uma das fontes supracitadas.

TABELA 1: ClassifiCAÇÕES E QUANTIDADES DE TRÍADES NAS FONTES DE DADOS DO EPINIONS, SLASHDOT E WIKIPÉDIA [14]. TRÍADE.

\begin{tabular}{cccc}
\hline \hline \multicolumn{2}{c}{ Tríade $T_{i}$} & $\left|T_{i}\right|$ & $p\left(T_{i}\right)$ \\
\hline \hline$T_{3}$ & +++ & 11.640 .257 & $87 \%$ \\
\hline$T_{2}$ & +-- & 947.855 & $7,1 \%$ \\
\hline$T_{1}$ & ++- & 698.023 & $5,2 \%$ \\
\hline$T_{0}$ & --- & 89.272 & $0,7 \%$ \\
\hline \hline
\end{tabular}

As tríades podem variar conforme o valor atribuído aos relacionamentos na rede social, conforme apresentado na Tabela 1 Para executar o algoritmo T-SWEETS nas bases supracitadas, o tamanho do caminho entre dois nós também foi limitado a 3 - tamanho esse limitado pela tríade.

\section{c. Resultados da Primeira Fase do Experimento}

De acordo com as associações ternárias e os valores positivos e negativos, alguns cenários para a inferência de relacionamentos de confiança podem ser representados. A Figura 2 apresenta estes cenários.

Leskovec [14] apresentou um estudo de algumas possibilidades para a construção de tríades, de acordo com os valores $\{-1,+1\}$. Neste estudo, consta que dado dois relacionamentos com valores de confiança quaisquer $(+1$ ou -1$)$, qual a probabilidade do terceiro ser positivo ou negativo. O relacionamento com a seta vermelha na Figura 2 representa este terceiro relacionamento. Este quadro de probabilidades pode ser visualizado na Tabela 2.

As probabilidades apresentadas na Tabela 2 foram utilizadas para realizar uma análise dos algoritmos objeto de estudo deste trabalho. Assim, para cada uma das configurações apresentadas (Figura 2 e Tabela 2), a Tabela 3 apresenta os resultados obtidos. 

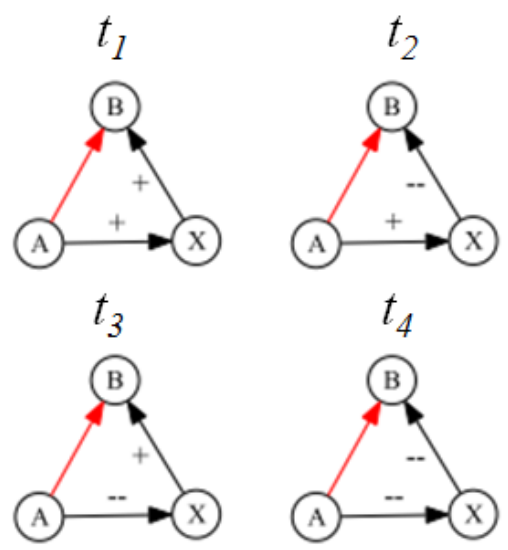

Figura 2: Possibilidades de tríades que podem ser usadas nas análises

TABELA 2: PRobabiLIDADES DE INFERÊNCIA DE RELACIONAMENTOS POSITIVOS E NEGATIVOS PARA CADA TRÍADE. ( [14] - ADAPTADA)

\begin{tabular}{ccc}
\hline \hline$t_{i}$ & $P(+)$ & $P(-)$ \\
\hline \hline 1 & 0,97 & 0,03 \\
\hline 2 & 0,54 & 0,46 \\
\hline 3 & 0,30 & 0,70 \\
\hline 4 & 0,23 & 0,77 \\
\hline \hline
\end{tabular}

Tabela 3: Porcentagem da Quantidade de RELACIONAMENTOS POSITIVOS EM CADA TRÍADE

\begin{tabular}{cccc}
\hline \hline \multirow{2}{*}{$t_{i}$} & & $P(+)$ & \\
& Leskovec [14] & TidalTrust & T-SWEETS \\
\hline \hline 4 & $97 \%$ & $100 \%$ & $100 \%$ \\
\hline 3 & $54 \%$ & $0 \%$ & $0 \%$ \\
\hline 2 & $30 \%$ & $0 \%$ & $0 \%$ \\
\hline 1 & $23 \%$ & $100 \%$ & $100 \%$ \\
\hline \hline
\end{tabular}

Conforme resultados apresentados na Tabela 3, tanto os resultados obtidos do T-SWEETS, quanto do TidalTrust, são valores muito determinísticos. Isto é, ou o grau de confiança foi nulo ou foi integral (100\%). Contudo, esta precisão pode ser justificável, uma vez que foram utilizados valores inteiros positivos e negativos. Para evitar esta limitação, o ideal é utilizar valores fracionados em uma escala de $[-1 \cdots+1]$.

\section{Limitação identificadas}

Leskovec [14] faz uso dessas tríades para analisar os seguintes princípios: "o amigo do meu amigo é meu amigo"; "o amigo do meu inimigo é meu inimigo"; "o inimigo do meu amigo é meu inimigo"; e "o inimigo do meu inimigo é meu amigo". Além disso, considera apenas valores inteiros de confiança, $\{-1,+1\}$. Essas características inviabilizam a utilização de um caminho no grafo social maior que 3 , já que tornaria ainda mais ineficaz a avaliação de confiança entre dois indivíduos. Essa ineficácia deve-se, principalmente, por tornar totalmente transitiva a relação de confiança entre dois nós $A$ e $B$, independente do tamanho do caminho entre eles. Esta ineficácia também ocorre em um caminho 3, porém. nestes casos, há uma representação dos princípios supraci- tados (por exemplo, o amigo do meu inimigo é meu inimigo) - o propósito de Leskovec. Tanto o T-SWEETS quanto o TidalTrust, realizam inferência com maior eficácia com entradas positivas fracionárias no intervalo $[0,1]$. Assim, é possível a obtenção de valores de confiança não discretos entre indivíduos da rede. Ou seja, a inferência de confiança não é totalmente transitiva, um dos princípios da análise de confiança.

\section{d. Resultados da Segunda Fase do Experimento}

Para realizar uma análise sobre valores fracionados e positivos, foi usado o conjunto de dados apresentados no trabalho de [9], que são usados para analisar o algoritmo Tidaltrust.

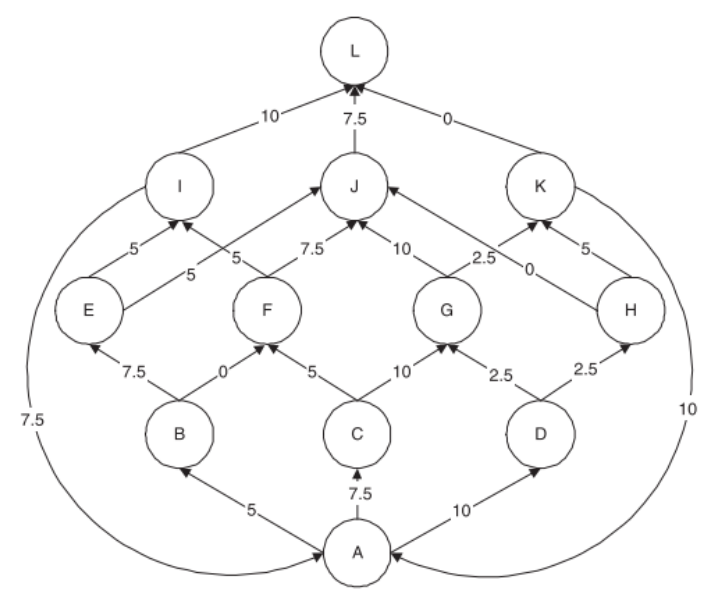

Figura 3: Grafo de relacionamentos utilizado para analisar os algoritmos ( [15])

Após a aplicação de ambos algoritmos, TidalTrust e TSWEETS, foram inferidos valores de confiança entre os nós apresentados no grafo da Figura 3. Com a aplicação dos algoritmos no cenário apresentado (Figura 3), o valor médio de confiança e o desvio padrão de ambos os algoritmos são apresentados na Tabela 4.

TABEla 4: MÉdia E DESVIO PADRÃo dos VALORES DOS QUADROS 5 E 6.

\begin{tabular}{ccc}
\hline \hline & Média & Desvio Padrão \\
\hline \hline T-SWEETS & 4,73 & 3,59 \\
\hline TidalTrust & 6,27 & 3,13 \\
\hline \hline
\end{tabular}

De acordo com o que pode ser observado na Tabela 4, a média dos valores de confiança entre os relacionamentos é maior no algoritmo TidalTrust. O valor menor obtido no TSWEETS é em decorrência de que este se baseia no princípio que quanto maior os caminhos de confiança que unem dois nós, menor é a relação de confiança entre eles. Assim, TSWEETS considera que pessoas que estejam mais próximas, têm maior tendência que haja uma maior relação de confiança entre elas, já que esta relação de confiança é baseada em informações (e.g. trocas de experiências) que as pessoas possuem umas sobre as outras.

Além da dispersão (existem pontos sobrepostos) dos valores obtidos com a execução dos algoritmos TidalTrust e TSWEETS, são aprestadas também as tendências dessas dispersão, considerando o aumento na distância do caminho so- 


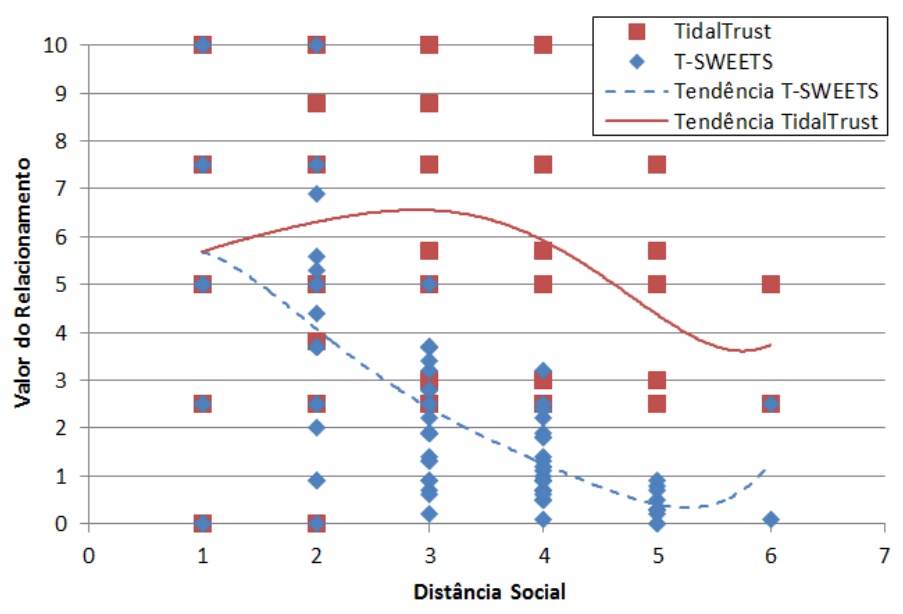

Figura 4: Dispersão dos valores obtidos para o grafo da Figura 3 com os algoritmos TidalTrust e T-SWEETS.

cial que liga dois nós no grafo. Essas linhas de tendências foram calculadas a partir da interpolação dos pontos na dispersão em um polinômio de grau 5. De acordo com o gráfico apresentado na Figura 4 (linha pontilhada) é possível observar que no T-SWEETS a tendência é decrescente, na maior parte de sua extensão, à medida que a distância social aumenta. Ao mesmo tempo, o algoritmo TidalTrust apresenta um comportamento sem padrão em relação à distância social. Diante da premissa que quanto maior a distância social entre dois nós há uma tendência de menor relação de confiança entre eles, o T-SWEETS apresenta um comportamento mais adequado.

\section{CONCLUSÕES}

A partir das investigações realizadas no presente trabalho, constatou-se que a abordagem T-SWEETS [13] considera com maior eficácia a relação entre o grau de confiança e a distância social em que duas pessoas estão na rede social, uma vez que tem uma tendência natural a diminuir o nível de confiança entre dois indivíduos se estes estão a uma distância social maior um do outro. Ao mesmo tempo, a abordagem TidalTrust [9] não possui um comportamento padrão considerando este afastamento entre as pessoas (a distância social entre elas).

Além disso, foi constatado também com esta análise que a utilização da escala que determina o grau de confiança entre dois indivíduos na rede social pode influenciar nos resultados das inferências dos relacionamentos de confiança que estão implícitos no ambiente. Assim, é sugerido que, em uma eventual implantação das abordagens em uma plataforma social, o ideal é que seja utilizada uma escala fracionada $[-1 \cdots+1]$ ao invés de valores inteiros positivos e negativos.

Como trabalho futuro, espera-se implantar os algoritmos de confiança apresentado no presente trabalho em momentos diferentes e verificar a eficácia de ambos em um ambiente real e, após, confrontar os resultados com os resultados obtidos no presente estudo. Além disso, espera-se analisar as demais características presentes no T-SWEETS e que também estejam presentes em outras abordagens disponíveis na literatura para identificar possíveis pontos fortes ou fragilidades existentes. Nesta perspectiva, espera-se obter análises que possam direcionar-nos a um formalismo ideal de um algoritmo para inferência de relacionamentos ocultos de confi- ança em ambientes virtuais sociais.

\section{REFERÊNCIAS}

[1] Y. Yamamoto, "A morality based on trust: Some reflections on japanese morality," 1990.

[2] T. Yamagishi and M. Yamagishi, "Trust and commitment in the united states and japan," Motivation and Emotion, vol. 18, pp. 129-166, 1994.

[3] T. Yamagishi, K. S. Cook, and M. Watabe, "Uncertainty, trust, and commitment formation in the united states and japan," 1998.

[4] H. Murayama, A. Taguchi, S. Ryu, S. Nagata, and S. Murashima, "Institutional trust in the national social security and municipal healthcare systems for the elderly in Japan," Health Promotion International, vol. 27, no. 3, pp. 394-404, 092011.

[5] F. J. McDonald, "Worlds of Truth: A Philosophy of Knowledge By Israel Scheffler," Analysis, vol. 70, no. 2, pp. 383-385, 022010.

[6] S. Marsh, Formalising Trust as a Computational Concept, ser. Technical report (University of Stirling. Department of Computing Science and Mathematics). University of Stirling, 1994.

[7] S. P. Marsh, "Formalising trust as a computational concept," Tech. Rep., 1994.

[8] S. Ries, J. Kangasharju, and M. Mühlhäuser, "A classification of trust systems," in On the Move to Meaningful Internet Systems 2006: OTM 2006 Workshops, R. Meersman, Z. Tari, and P. Herrero, Eds. Berlin, Heidelberg: Springer Berlin Heidelberg, 2006, pp. 894-903.

[9] J. Golbeck, "Computing and applying trust in web-based social networks," Ph.D. dissertation, 2005.

[10] M. Taherian, M. Amini, and R. Jalili, "Trust inference in web-based social networks using resistive networks," in 2008 Third International Conference on Internet and Web Applications and Services, June 2008, pp. 233-238.

[11] U. Kuter, "Sunny: A new algorithm for trust inference in social networks using probabilistic confidence models," in In Proceedings of the National Conference on Artificial Intelligence (AAAI, 2007.

[12] U. Kuter and J. Golbeck, "Using probabilistic confidence models for trust inference in web-based social networks," ACM Trans. Internet Technol., vol. 10, no. 2, Jun. 2010. [Online]. Available: https://doi.org/10.1145/1754393.1754397

[13] E. M. Silva, D. O. Rodrigues, J. G. Souza, P. F. Brito, A. C. Salgado, S. R. Meira, and J. A. Costa, "Inferring hidden trust relationships in social networks for encouraging collaboration and cooperation among individuals," in Proceedings of the 19th International Conference on Collaboration and Technology - Volume 8224. Berlin, Heidelberg: Springer-Verlag, 2013, p. 42-60.

[14] J. Leskovec, D. Huttenlocher, and J. Kleinberg, "Signed networks in social media," in Proceedings of the SIGCHI Conference on Human Factors in Computing Systems, ser. CHI '10. New York, NY, USA: Association for Computing Machinery, 2010, p. 1361-1370. 
[15] M. Taherian, M. Amini, and R. Jalili, "Trust inference in web-based social networks using resistive networks," 2008 Third International Conference on Internet and Web Applications and Services, pp. 233238, 2008. 